\title{
DESERCIÓN ESTUDIANTIL EN COLOMBIA Y LOS PROGRAMAS DE INGENIERÍA DE LA UPTC SECCIONAL SOGAMOSO
}

\section{Student dropout in Colombia and the engineering programs of the Uptc campus Sogamoso}

\author{
Oscar Iván Higuera Martínez ${ }^{1}$ \\ ${ }^{1}$ Universidad Pedagógica y Tecnológica de Colombia, Escuela de Ingeniería Electrónica, Grupo de \\ Investigación DSP UPTC, Colombia. Email: oscar.higuera@uptc.edu.co
}

(Recibido noviembre 15 de 2016 y aceptado diciembre 6 de 2016)

\begin{abstract}
Resumen
Este artículo presenta un análisis de la deserción estudiantil en Colombia, inicialmente en las áreas de programas que posee la Uptc Sogamoso y finalmente enfocándose en los programas de Ingeniería de la sede, donde se revisan los datos reportados por SPADIES (Sistema para la Prevención de la Deserción en las Instituciones de Educación superior), y se realiza una descripción de los resultados reportados y algunas variables que influyen en la deserción en la Uptc Sogamoso.
\end{abstract}

Palabras clave: Deserción estudiantil, educación superior, ingeniería.

\begin{abstract}
This paper presents an analysis of student dropout in Colombia. Initially in the programs areas that Uptc Sogamoso has and finally focusing on the Engineering programs in the campus, where the data reported by SPADIES (System for the Prevention of Dropout in Institutions of Higher Education) are reviewed, and a description is made of the results reported and the some variables that influence the dropout in the Uptc Sogamoso.
\end{abstract}

Key words: Student dropout, higher education, engineering.

\section{INTRODUCCIÓN}

El análisis de las causas de deserción es una herramienta importante al momento de determinar las acciones que deben realizarse para aumentar la retención y graduación de los estudiantes en la educación superior en Colombia. El análisis de deserción y sus principales consecuencias es un trabajo que se realiza desde hace décadas en Colombia y Latinoamérica, muestra de ello son trabajos como [1], donde se presenta la deserción estudiantil en la educación superior de Colombia, una metodología de seguimiento, diagnóstico y elementos para su prevención, documento elaborado por el Ministerio de Educación Nacional de Colombia, y donde se brinda información sobre la situación a 2009 y presenta elementos útiles para el análisis y tratamiento de la deserción estudiantil en educación superior, a partir de una metodología implementada en el Sistema de Prevención de la Deserción en Educación Superior (SPADIES), en [2] se analiza las interpretaciones sobre la deserción en la universidad colombiana. Las miradas sobre deserción se orientan a reflexionar y ofrecer mecanismos de intervención en las universidades, sin relacionarlo con la disminución del valor social de la educación superior.

En [3] se realizó una revisión sistemática para precisar las características del estrés académico que incide en la salud mental de la población universitaria. Donde se examinaron publicaciones sobre estrés académico, deserción estudiantil y estrategias de retención. Así como universidades que han realizado análisis a sus campus y programas como en [4], donde la Universidad de Antioquia presenta un análisis del problema en Colombia y un panorama general del fenómeno de la deserción en la Universidad de Antioquia, y se realiza un análisis descriptivo de las características sociodemográficas de la población. Otras Universidades que han realizado investigaciones sobre este tema son [5-10] donde se exploran desde conceptos de deserción, factores de 
riesgo presentes, como casos de estudio puntual sobre la deserción en las diferentes instituciones de educación superior o programas analizados y formular variables a considerar para un adecuado seguimiento y diagnóstico.

Al momento de analizar los diferentes tipos de deserción, una de las características que más se observa es la determinada con respecto al tiempo, donde la deserción se divide en [11]: Deserción precoz: el individuo que, habiendo sido aceptado por la universidad, no se matricula; deserción temprana: aquel que abandona sus estudios en los primeros semestres de la carrera, en esta etapa es donde más se concentra la deserción, la cual está en el orden de un 75\%; y deserción tardía: quien abandona los estudios en los últimos semestres, es decir, una vez cursados al menos la mitad de los semestres establecidos en el programa académico.

El Ministerio de Educación Nacional de Colombia creó El Sistema de Prevención de la Deserción en Educación Superior, SPADIES, que según el MEN "Es la herramienta para hacer seguimiento sobre las cifras de deserción de estudiantes de la educación superior. Con los datos suministrados por las instituciones de educación superior a SPADIES, se identifican y se ponderan los comportamientos, las causas, variables y riesgos determinantes para desertar. Además, con esta información se agrupan los estudiantes de acuerdo con su riesgo de deserción", el cual está disponible en internet [15], es por esto que este sistema sirve y da información para analizar la deserción en cada institución de educación superior y poder tomar acciones al respecto, actualmente este sistema está instalado en el $99 \%$ de las Instituciones de educación superior y hace seguimiento a más de 3 millones de estudiantes.

\section{DESERCIÓN NACIONAL}

En el análisis de deserción nacional, se puede observar que en la Educación superior según lo muestran las estadísticas de SPADIES la mayor deserción se encuentra en los programas técnico y tecnológico, los cuales llegan al $62.18 \%$ y $59.53 \%$ respectivamente, y para la Educación Universitaria es de $44,93 \%$ en el 10 semestre, en la figura 1 se observa este comportamiento. Estos valores son similares a los reportados en el año 2013, donde la deserción en el nivel universitario alcanzó el $44.9 \%$, y para el nivel técnico y tecnológico la deserción alcanzó niveles del $62.4 \%$ y el $53.8 \%$ respectivamente [12]. Lo cual hace reflexionar sobre ¿Que está pasando con las políticas de disminución de la deserción estudiantil a nivel nacional? Pero adicional a estos datos, es de preocuparse que con respecto al origen de la institución, la deserción en las instituciones de carácter oficial en 10 semestre está en $49.03 \%$, mientras las no oficiales están en $49.21 \%$, y donde el promedio de la deserción por cohorte, periodo $1998-2007$ estaba en $47 \%$ para IES públicas y en $46 \%$ para IES privadas [14].

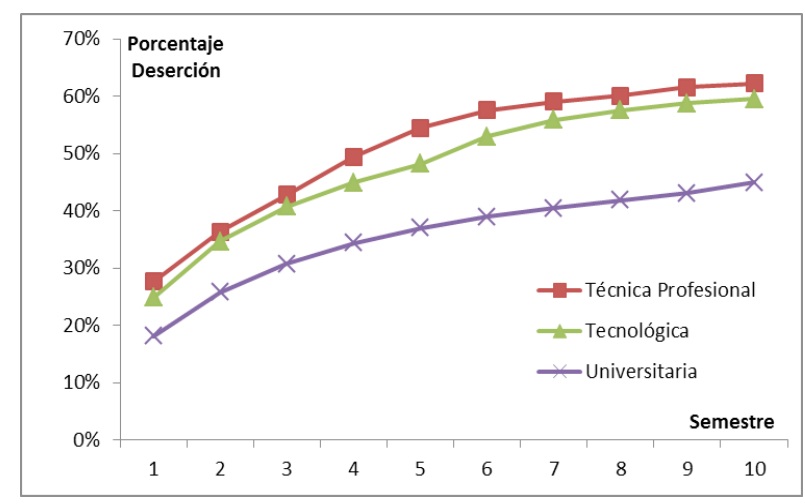

Figura 1. Estadísticas de deserción en educación superior por nivel de formación, [15].

Con respecto a la deserción en el departamento de Boyacá, y según los datos proporcionados por SPADIES, se logra observar que en el año 2008 era de $38.2 \%$ [13] y en el 2013 de $43.2 \%$ [12] y el actual (consulta en noviembre 15 de 2016) es de $45.14 \%$, reportando un crecimiento proporcional al Nacional, lo cual indica que en Boyacá este índice también está en aumento, que si bien Boyacá está ubicado en la posición 9 de los índices de deserción más bajos, se debe revisar si los procesos que se están realizando en las universidades del departamento están siendo bien enfocados, en donde las instituciones oficiales reportan una deserción de $44.44 \%$, mientras que las no oficiales de $46,77 \%$.

En la Tabla 1 se observan las estadísticas de deserción nacional según diferentes criterios como ingreso de la familia del estudiante (IFE), clasificación examen de estado (CEE), trabajaba al presentar el ICFES (TPI) y el nivel educativo de la madre (NEM), donde como se esperaba, las características al momento de ingreso del estudiante al sistema de educación superior es influyente en la deserción, observando que se presenta menor deserción entre mayor es el ingreso del grupo familiar, y si esté trabaja, lo cual incluye para consideración de parte de las IES una posible consecuencia de deserción no académica, así mismo el resultado de las pruebas de es- 
tado determina que los estudiantes clasificados en bajo, tienen un riego (20\%) mayor a los clasificados en alto, lo cual introduce un riesgo académico de deserción para estos.

Tabla 1. Deserción a 10 semestres considerando factores socio-económicos y de ingreso a la IES.

\begin{tabular}{clc}
\hline & Aspecto Considerado & Deserción \\
\hline \multirow{2}{*}{ IFE } & {$[0,1)$ salarios mínimos } & $52.87 \%$ \\
& {$[1,2)$ salarios mínimos } & $51.86 \%$ \\
& {$[2,3)$ salarios mínimos } & $49.45 \%$ \\
& {$[3,5)$ salarios mínimos salarios mínimos } & $45.79 \%$ \\
& {$[7,10)$ salarios mínimos } & $42.18 \%$ \\
& {$[10$,$) salarios mínimos$} & $42.76 \%$ \\
\multirow{2}{*}{ CEE } & Bajo & $38.67 \%$ \\
\hline \multirow{2}{*}{ TPI } & Medio & $57.20 \%$ \\
& No trabajaba & $47.24 \%$ \\
& Si trabajaba & $37.20 \%$ \\
\hline \multirow{2}{*}{ Básica primaria o inferior } & $48.24 \%$ \\
& Básica secundaria & $57.13 \%$ \\
\hline \multirow{2}{*}{ NEM } & Media vocacional o técnica/ & $53.05 \%$ \\
& tecnológica & $50.97 \%$ \\
& Universitaria o superior & $42.58 \%$ \\
\hline
\end{tabular}

[15]

\section{DESERCIÓN EN LAS ÁREAS DE INGENIERÍA Y CIENCIAS ECONÓMICAS Y ADMINISTRATIVAS}

En cuanto a la deserción considerando las diferentes áreas de conocimiento, se observa que la más elevada se encuentra en el área de ingeniería, arquitectura, urbanismo y afines con un 54,87\%, (Ver tabla 2), pero a su vez en esta área se observa que la educación técnica profesional con un $73.17 \%$ es la más alta seguida de la tecnológica con un $64.41 \%$ y la universitaria con un $48.82 \%$. Para el interés de este texto se considera la deserción en las áreas de ingeniería, arquitectura, urbanismo y afines, y en economía, administración, contaduría y afines, por cuanto son en las áreas que se imparten los programas de pregrado en la Uptc Seccional Sogamoso. Tomando a nivel nacional en el nivel universitario en las áreas de interés, la deserción esta para economía, administración, contaduría y afines en $44.26 \%$ y en ingeniería, arquitectura, urbanismo y afines de $48.82 \%$.
Tabla 2. Deserción por área de conocimiento.

\begin{tabular}{|c|c|c|c|c|}
\hline $\begin{array}{c}\text { Área de } \\
\text { conocimiento }\end{array}$ & Sem 2 & Sem 4 & Sem 6 & Sem10 \\
\hline $\begin{array}{l}\text { Agronomía, } \\
\text { veterinaria y } \\
\text { afines }\end{array}$ & $33.25 \%$ & $42.99 \%$ & $49.02 \%$ & $54.38 \%$ \\
\hline Bellas artes & $27.03 \%$ & $36.73 \%$ & $43.38 \%$ & $49.56 \%$ \\
\hline $\begin{array}{l}\text { Ciencias de la } \\
\text { educación }\end{array}$ & $26.77 \%$ & $35.01 \%$ & $39.61 \%$ & $44.93 \%$ \\
\hline $\begin{array}{l}\text { Ciencias de la } \\
\text { salud }\end{array}$ & $22.86 \%$ & $30.19 \%$ & $34.66 \%$ & $40.02 \%$ \\
\hline $\begin{array}{l}\text { Ciencias } \\
\text { sociales y } \\
\text { humanas }\end{array}$ & $23.78 \%$ & $31.29 \%$ & $35.48 \%$ & $42.44 \%$ \\
\hline $\begin{array}{l}\text { Economía, } \\
\text { administración, } \\
\text { contaduría y } \\
\text { afines }\end{array}$ & $29.87 \%$ & $39.02 \%$ & $44.52 \%$ & $49.93 \%$ \\
\hline $\begin{array}{l}\text { Ingeniería, } \\
\text { arquitectura, } \\
\text { urbanismo y } \\
\text { afines }\end{array}$ & $31.90 \%$ & $42.42 \%$ & $48.79 \%$ & $54.87 \%$ \\
\hline $\begin{array}{l}\text { Matemáticas } \\
\text { y ciencias } \\
\text { naturales }\end{array}$ & $30.98 \%$ & $40.91 \%$ & $45.97 \%$ & $50.56 \%$ \\
\hline
\end{tabular}

Las Instituciones oficiales presentan una mayor deserción solo considerando la Educación universitaria en las áreas de Economía, administración, contaduría y afines con $45.05 \%$ y en ingeniería, arquitectura, urbanismo y afines con $50.85 \%$, mientras las no oficiales en economía, administración, contaduría y afines con $43.79 \%$ y en ingeniería, arquitectura, urbanismo y afines con $47.22 \%$.

Considerando solo el departamento de Boyacá y el nivel de educación universitaria, en el área de ciencias económicas, el departamento se encuentra en el puesto 10 de 26 con una deserción por cohorte de 40,93, y en ingeniería de 7 de 27 con $44,32 \%$, mostrando bajos indicadores de deserción en comparación con otros departamentos. En la Tabla 3 se puede apreciar la deserción por cohorte en décimo semestre en las diferentes sedes de la Uptc para las áreas de economía, administración, contaduría y afines, e ingeniería, arquitectura, urbanismo y afines, se observa que la mayor deserción en estas áreas se presenta en Sogamoso. 
Tabla 3. Deserción en décimo semestre en la sedes de la Uptc para las áreas de economía, administración, contaduría y afines, e ingeniería, arquitectura, urbanismo y afines.

\begin{tabular}{ccc}
\hline Área de conocimiento & Sede & Des. \\
\hline \multirow{2}{*}{$\begin{array}{c}\text { Economía, administración, } \\
\text { contaduría y afines }\end{array}$} & Duitama & $32.82 \%$ \\
& Chiquinquirá & $44.16 \%$ \\
& Sogamoso & $45.04 \%$ \\
\hline \multirow{2}{*}{ Ingeniería, arquitectura, } & Tunja & $42.93 \%$ \\
urbanismo y afines & Duitama & $45.09 \%$ \\
& Sogamoso & $45.90 \%$ \\
\hline
\end{tabular}

[15]

\section{DESERCIÓN EN LOS PROGRAMAS DE INGENIERÍA DE LA UPTC SOGAMOSO}

En esta sección se enfoca el análisis en la deserción en los programas de la Seccional Sogamoso de la Uptc, iniciando por una mirada general y enfocándose el análisis en los programas de ingeniería de la misma.

Como se observó en la tabla 3, la deserción en las áreas de economía, administración, contaduría y afines, está en 45,04 , y en ingeniería, arquitectura, urbanismo y afines en 45,90 , los cuales son inferiores a la deserción para las mismas áreas a nivel nacional, En la Figura 2 podemos detallar que el programa de ingeniería electrónica, posee la más alta deserción y los programas de ingeniería industrial y contaduría pública la menor deserción, y analizando todos los programas se logra determinar que en promedio en los 4 primeros semestres se tiene una deserción del $72,6 \%$ de la total generada hasta décimo semestre, que es la denominada deserción temprana, que es donde se ha concentrado el esfuerzo de reducir la deserción en la Uptc.

En las Figura 3 y 4 se puede observar la deserción por periodo, donde se está presentando una tendencia a la baja en la deserción por periodo académico, y esto es debido a las estrategias implementadas por la universidad para reducir la deserción, entre las cuales se tienen de tipo académicas, donde se rescata el plan padrino, el cual en la Sede es orientado por los docentes de las diferentes áreas donde se ha identificado debilidades por parte de los estudiantes, y los cursos intersemestrales. Y de tipo no académicas, donde se rescata la implementa- ción desde hace algunos años de las becas por extrema incapacidad, residencias estudiantiles, becas deportivas, culturales y de trabajo en dependencias de la universidad, las cuales buscan ayudar a los estudiantes en la parte económica.

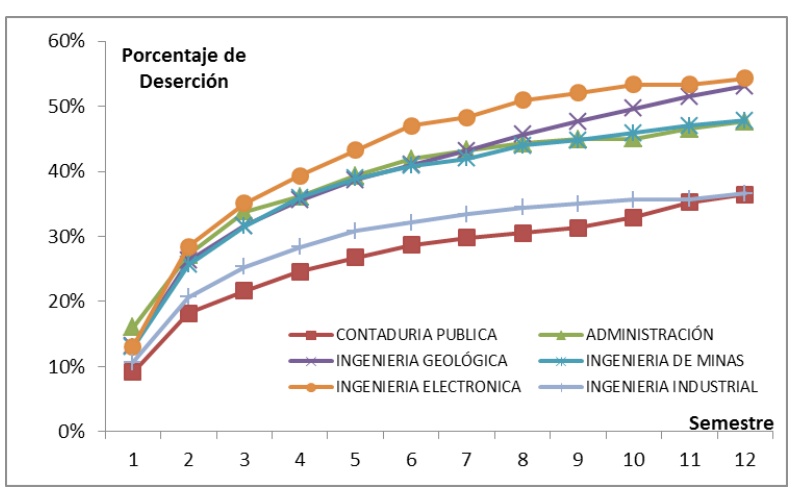

Figura 2. Estadísticas de deserción por cohorte programas Uptc Sogamoso, [15].

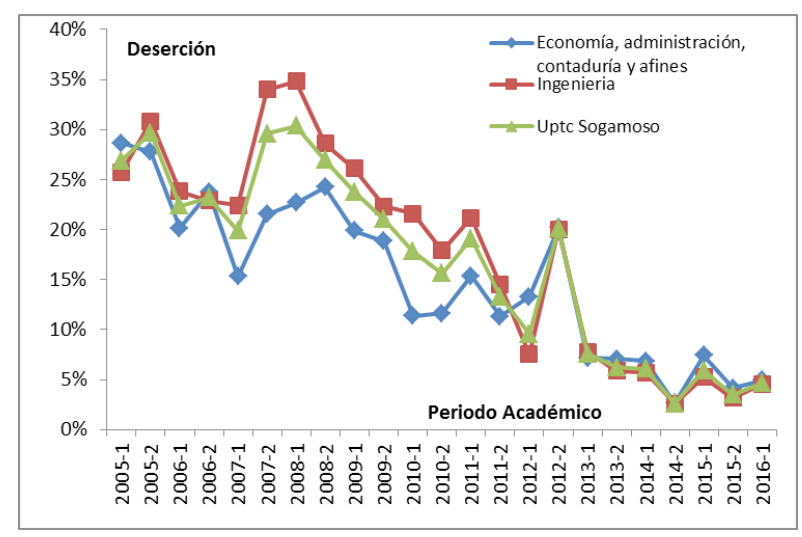

Figura 3. Estadísticas de deserción por periodo por área de conocimiento Uptc Sogamoso, [15].

Finalmente revisando la deserción en los programas de Ingeniería Uptc Sogamoso, con los programas de Ingeniería que están adscritos en el SNIES en la Uptc Sogamoso (código institución 1108), en la deserción por cohorte, como se mencionó anteriormente se tiene un comportamiento muy parecido en los programas de ingeniería de la seccional con respecto al área de ingeniería en Colombia, a excepción de ingeniería industrial, quien tiene una deserción inferior a los demás programas (Figura 5). 


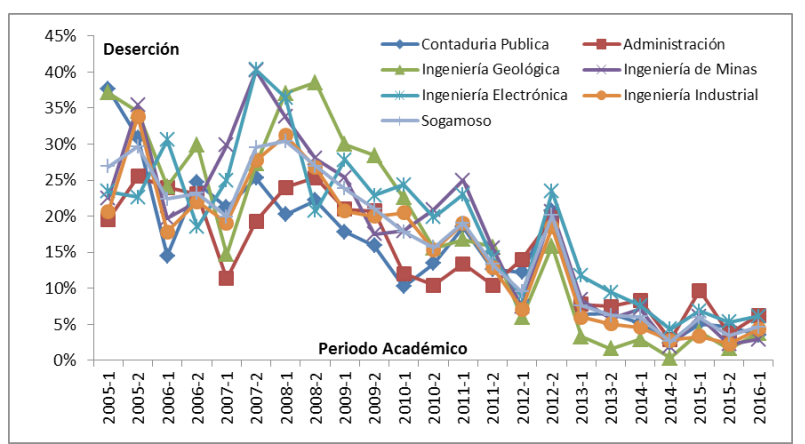

Figura 4. Estadísticas de deserción por periodo por programa académico Uptc Sogamoso, [15].

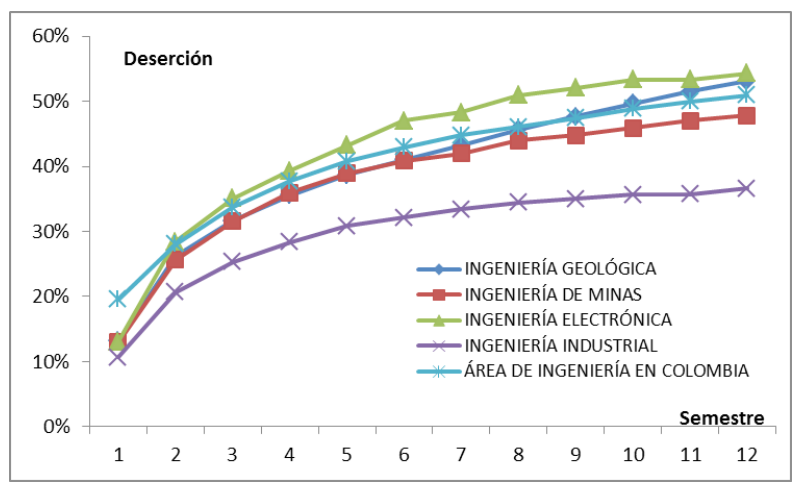

Figura 5. Estadísticas de deserción por cohorte en los Programas de Ingeniería Uptc Sogamoso, [15].

En las Tablas 4, y 5, se observa la clasificación según algunas características socioeconómicas y de ingreso a la universidad para los programas de ingeniería de la sede. Donde se observa que los principales factores que están influyendo en la deserción y que deben ser considerados al momento de realizar un seguimiento con el fin de disminuir la deserción según los datos reportados en el SPADIES son:

En cuanto a la deserción por presentación de la prueba de estado, se encuentra que existe una mayor deserción cuando el estudiante trabajaba al momento de presentar la prueba, lo que implica una deserción no académica potencial a futuro, mientras que en cuanto al resultado de la prueba, se observa que entre mejor resultado es menor la deserción que se produce, lo que implica un potencial desertor si el resultado de la prueba es baja.

En cuanto a ingreso del núcleo familiar (Tabla 4), no se puede llegar a concluir por cuanto a diferencia de lo que sucede a nivel nacional, donde se observa que entre menor ingreso mayor deserción, en los programas de ingeniería, no se puede concluir lo mismo, por cuanto la deserción es distribuida sin sesgo hacia uno de los dos lados excepto ingeniero industrial, donde la deserción es mayor si el ingreso del núcleo familiar es mayor, Analizando otra variable como lo es nivel educativo de la madre, sucede algo similar a lo presentado con los ingresos familiares, donde la distribución de deserción no puede ser concluyente al respecto.

Tabla 4. Deserción en decimo semestre considerando el Ingreso de la familia del estudiante

\begin{tabular}{ccc}
\hline Programa & $\begin{array}{c}\text { Ingreso en Salarios } \\
\text { Mínimos }\end{array}$ & Deserción \\
\hline Ingeniería & {$[0,1)$} & $51.35 \%$ \\
Geológica & {$[1,2)$} & $47.62 \%$ \\
& {$[2,3)$} & $59.33 \%$ \\
& {$[3,5)$} & $50.00 \%$ \\
& {$[5,7)$} & $0.00 \%$ \\
\hline Ingeniería de & {$[0,1)$} & $39.87 \%$ \\
Minas & {$[1,2)$} & $50.29 \%$ \\
& {$[2,3)$} & $41.44 \%$ \\
& {$[3,5)$} & $43.84 \%$ \\
& {$[5,7)$} & $37.50 \%$ \\
Ingeniería & {$[7,10)$} & $45.45 \%$ \\
\hline Electrónica & {$[0,1)$} & $59.26 \%$ \\
& {$[1,2)$} & $54.61 \%$ \\
& {$[2,3)$} & $52.69 \%$ \\
\hline & {$[3,5)$} & $62.71 \%$ \\
& {$[5,7)$} & $57.89 \%$ \\
\hline Ingeniería & {$[0,1)$} & $29.60 \%$ \\
Industrial & {$[1,2)$} & $33.04 \%$ \\
& {$[2,3)$} & $34.91 \%$ \\
& {$[3,5)$} & $42.17 \%$ \\
& {$[5,7)$} & $55.56 \%$ \\
\hline
\end{tabular}

[15]

\section{CONCLUSIONES}

De esta revisión se puede concluir que aunque la Uptc Sogamoso tiene estrategias implementadas para disminuir la deserción, lo que se ve representado en la disminución de la deserción por cohorte, se debe intensificar el esfuerzo en realizar un seguimiento a los estudiantes desde cuando ingresan y de esta forma disminuir la de- 
serción en los diferentes programas, así como identificar las variables preponderantes en la deserción.

Se sugiere implementar una entrevista personalizada, con el fin de identificar desde el inicio que estudiantes tienen problemas económicos con el fin de disminuir la deserción no académica basada en los ingresos de la familia y reforzar el plan padrino para los estudiantes que ingresan a primeros semestres.

Tabla 5. Deserción en decimo semestre considerando el Examen de Estado

\begin{tabular}{|c|c|c|}
\hline Programa & $\begin{array}{c}\text { Trabajaba al } \\
\text { presentar }\end{array}$ & Deserción \\
\hline \multirow{2}{*}{ Ingeniería de Minas } & No & $44.56 \%$ \\
\hline & $\mathrm{Si}$ & $65.12 \%$ \\
\hline \multirow{2}{*}{ Ingeniería Electrónica } & No & $53.02 \%$ \\
\hline & $\mathrm{Si}$ & $60.00 \%$ \\
\hline \multirow{2}{*}{ Ingeniería Geológica } & No & $48.93 \%$ \\
\hline & $\mathrm{Si}$ & $70.21 \%$ \\
\hline \multirow{2}{*}{ Ingeniería Industrial } & No & $34.84 \%$ \\
\hline & $\mathrm{Si}$ & $40.54 \%$ \\
\hline Programa & Clasificación & Deserción \\
\hline \multirow{3}{*}{ Ingeniería de Minas } & Alto & $35.46 \%$ \\
\hline & Medio & $47.97 \%$ \\
\hline & Bajo & $62.60 \%$ \\
\hline \multirow{3}{*}{ Ingeniería Electrónica } & Alto & $47.42 \%$ \\
\hline & Medio & $60.60 \%$ \\
\hline & Bajo & $69.74 \%$ \\
\hline \multirow{3}{*}{ Ingeniería Geológica } & Alto & $42.34 \%$ \\
\hline & Medio & $48.69 \%$ \\
\hline & Bajo & $65.38 \%$ \\
\hline \multirow{3}{*}{ Ingeniería Industrial } & Alto & $34.66 \%$ \\
\hline & Medio & $31.69 \%$ \\
\hline & Bajo & $52.08 \%$ \\
\hline
\end{tabular}

[15]

\section{REFERENCIAS}

[1] Ministerio de Educación Nacional de Colombia (2009), Deserción estudiantil en la educación superior Colombiana Metodología de seguimiento, diagnóstico y elementos para su prevención.

[2] Barragán-Diaz, D. Patiño-Garzón, L (2013) Elementos para la comprensión del fenómeno de la de- serción universitaria en Colombia. Más allá de las mediciones. Cuadernos Latinoamericanos de Administración Vol IX Número 16. Págs. 55-66. DOI: http://dx.doi.org/10.18270/cuaderlam.v9i16.1248

[3] Suárez-Montes, N. \& Díaz-Subieta, L. (2015). Estrés académico, deserción y estrategias de retención de estudiantes en la educación superior. Revista de Salud Pública, Vol 17 Numero 2, 300-313. DOI: https://dx.doi.org/10.15446/rsap.v17n2.52891

[4] Vásquez-Velásquez, J. Castaño-Vélez, E. gGallón-Gómez, S. y Gómez-Portilla, K. (2003) Determinantes de la desercion estudiantil en la Universidad de Antioquia. Informe Final de Investigación Centro de Investigaciones Económicas - CIE- de la Universidad de Antioquia.

[5] Londoño Ardila, L. (2013) Factores de riesgo presentes en la deserción estudiantil en la Corporación Universitaria Lasallista, Revista Virtual Universidad Católica del Norte, No 38. pp 183- 194.

[6] Patiño-Garzón, L. y Cardona-Pérez, A. (2012) Revisión de algunos estudios sobre la deserción estudiantil universitaria en Colombia y latinoamérica. Theoria, Vol. 21 (1). Pp 9-20.

[7] González Pineda, C. Beltran Ortega, H. (2015) Deserción estudiantil en la universidad pública Colombiana: programa de economía de la Universidad de Cartagena. Trabajo de grado para optar por el título de Economista, Universidad de Cartagena

[8] Guzman Puentes, S. (2009) Deserción y Retención Estudiantil en los Programas de pregrado de la Pontificia Universidad Javeriana. Informe final de Investigación para optar el Titulo de Magister en Educacion.

[9] Chinome-Becerra, P. Ruiz-Cardenas, C. Fernandez-Samacá, L. (2016) Priorización de variables en el diseño de un sistema de gestión integral de la deserción estudiantil Revista Educación en Ingenieria. Vol. 11, Num. 22 pp 6977.

[10] OECD Publishing, (2016) Reviews of National Policies for Education Education in Colombia, https:// books.google.com.co/books?id=oB8EDAAAQBAJ

[11] Castaño, E., Gallón, S., Gomez, K. y Vasquez, J., (2008) Análisis de los factores asociados a la deserción estudiantil en la educación superior: un estudio de caso, Revista de Educación, Vol 1 No 345, pp 255-280.

[12] Ministerio de Educacion Nacional de Colombia (2014). "Tasa de desercion acumulada por cohorte según semestre, SPADIES". Informe MEN Disponible en: http://www.mineducacion.gov.co/ 
sistemasdeinformacion/1735/articles-254702_archivo_pdf_estadisticas_2013.pdf

[13] Ministerio de Educacion Nacional de Colombia (2010). "Boletin de Educacion superior No 14". Disponible en http://www.mineducacion.gov. co/1759/articles-92779_archivo_pdf_Boletin14. pdf
[14] Ministerio de Educacion Nacional de Colombia (2009). Deserción Estudiantil en la Educacion superior Colombiana. Disponible en http://www.mineducacion.gov.co/sistemasdeinformacion/1735/ articles-254702_diagnostico_desercion.pdf

[15] Ministerio de Educacion Nacional, (2016) SPADIES, Disponible en http://spadies.mineducacion.gov. co/spadies/JSON.html Consultado Nov 15 de 2016 